\title{
Perfil dos transtornos de ansiedade e fatores associados em universitários de um centro universitário de Teresina, Piauí
}

\author{
Profile of anxiety disorders and associated factors in university students at a university center in \\ Teresina, Piauí
}

Perfil de los trastornos de ansiedad y factores asociados en estudiantes universitarios de un centro universitario de Teresina, Piauí

\author{
Reinaldo de Brito Santos \\ ORCID: https://orcid.org/0000-0002-7327-6521 \\ Centro Universitário Santo Agostinho, Brasil \\ E-mail: reinaldosantos.018@hotmail.com \\ Guilherme Viana Soares Feitosa \\ ORCID: https://orcid.org/0000-0002-5639-4892 \\ Centro Universitário Santo Agostinho, Brasil \\ E-mail: guiiviana000@gmail.com \\ Leyla Régis de Meneses Carvalho Sousa Carvalho \\ ORCID: https://orcid.org/0000-0001-9912-4166 \\ Centro Universitário Santo Agostinho, Brasil \\ E-mail: leyla.regis@hotmail.com
}

\begin{abstract}
Resumo
O ingresso no ensino superior e a vivência acadêmica caracterizam um marco significativo que coincide a um período do desenvolvimento psicossocial marcado por mudanças importantes, como o período de explorações, a idade das possibilidades e instabilidades. O estudo objetiva traçar o perfil dos níveis de ansiedade em estudantes universitários de um Centro Universitário e caracterizar a ansiedade e fatores associados destes acadêmicos da cidade de Teresina, Piauí. Trata-se de um estudo com análise quantitativa descritiva, com amostra composta por 165 sujeitos escolhidos por conveniência. Os critérios de inclusão: alunos regularmente matriculados na instituição; ter idade entre 18 anos e 40 anos; independente da raça e classe social e que consentiram em participar da pesquisa. Através da análise estatística, os níveis de ansiedade: $4(2,4 \%)$ apresentaram a classificação mínima; 9 apresentaram nível leve, sendo 7 $(4,2 \%)$ do sexo masculino e $2(1,3 \%)$ do sexo feminino; 48 apresentaram nível moderado, sendo 26 (15,8\%) do sexo masculino e $22(13,3 \%)$ do sexo feminino e 104 apresentaram nível considerado grave, sendo 50 (30,3\%) do sexo masculino e $54(32,7 \%)$ do sexo feminino. Verificou-se que a maioria dos universitários apresentaram níveis graves de ansiedade, observando discreta maioria do índice grave relacionado ao sexo feminino. Os níveis de ansiedade prevaleceram no sexo feminino que não aderem a atividade física regular. Contudo, deve-se repensar sobre o processo de formação desde o ingresso até a sua conclusão, buscar por uma formação assistida, aonde o estudante seja atendido em suas necessidades pedagógicas e emocionais.
\end{abstract}

Palavras-chave: Ansiedade; Transtornos de ansiedade; Universitários.

\begin{abstract}
Entry into higher education and academic experience characterize a significant milestone that coincides with a period of psychosocial development marked by important changes, such as the period of exploration, the age of possibilities and instabilities. The study aims to outline the profile of anxiety levels in university students at a University Center and to characterize the anxiety and associated factors of these academics in the city of Teresina, Piauí. This is a study with descriptive quantitative analysis, with a sample composed of 165 subjects chosen for convenience. Inclusion criteria: students regularly enrolled at the institution; be between 18 and 40 years old; regardless of race and social class and who consented to participate in the research. Through statistical analysis, the levels of anxiety: 4 (2.4\%) had the minimum classification; 9 had a mild level, with $7(4.2 \%)$ being male and $2(1.3 \%)$ being female; 48 had a moderate level, $26(15.8 \%)$ were male and $22(13.3 \%)$ were female and 104 had a level considered severe, $50(30.3 \%)$ were male and $54(32,7 \%)$ female. It was found that the majority of university students had severe levels of anxiety, observing a slight majority of the serious index related to the female sex. Anxiety levels prevailed in females who do not adhere to regular physical activity. However, it is necessary to rethink the training process from admission to its conclusion, to seek assisted training, where the student is attended to in his pedagogical and emotional needs.
\end{abstract}

Keywords: Anxiety; Anxiety disorders; College students. 


\begin{abstract}
Resumen
El ingreso a la educación superior y la experiencia académica caracterizan un hito significativo que coincide con un período de desarrollo psicosocial marcado por cambios importantes, como el período de exploración, la era de las posibilidades y las inestabilidades. El estudio tiene como objetivo perfilar el perfil de los niveles de ansiedad en estudiantes universitarios de un Centro Universitario y caracterizar la ansiedad y factores asociados de estos académicos en la ciudad de Teresina, Piauí. Se trata de un estudio con análisis cuantitativo descriptivo, con una muestra compuesta por 165 sujetos elegidos por conveniencia. Criterios de inclusión: estudiantes matriculados regularmente en la institución; tener entre 18 y 40 años; independientemente de su raza y clase social y de quién consintió en participar en la investigación. Mediante análisis estadístico, los niveles de ansiedad: 4 (2,4\%) tuvieron la clasificación mínima; 9 tenían un nivel leve, siendo 7 (4,2\%) hombres y 2 (1,3\%) mujeres; 48 tenían un nivel moderado, $26(15,8 \%)$ eran hombres y $22(13,3 \%)$ eran mujeres y 104 tenían un nivel considerado severo, 50 (30,3\%) eran hombres y $54(32,7 \%)$ mujeres. Se encontró que la mayoría de los estudiantes universitarios presentaba niveles severos de ansiedad, observándose una ligera mayoría del índice grave relacionado con el sexo femenino. Los niveles de ansiedad prevalecieron en las mujeres que no se adhieren a la actividad física regular. Sin embargo, es necesario repensar el proceso formativo desde el ingreso hasta su conclusión, para buscar una formación asistida, donde se atienda al alumno en sus necesidades pedagógicas y emocionales.
\end{abstract}

Palabras clave: Ansiedad; Desórdenes de ansiedad; Estudiantes universitarios.

\title{
1. Introdução
}

Segundo dados da Organização Mundial de Saúde (2017), a prevalência mundial do Transtorno de Ansiedade (TA) é de 3,6\%. No continente americano esse transtorno mental alcança maiores proporções, sendo de 5,6\% da população, com evidência para o Brasil, onde está presente em 9,3\% da população, possuindo o maior número de casos dentre todos os países do mundo.

A ansiedade representa uma situação frequente e cotidiana nos estudantes universitários, sendo conceituada como um estado de diferentes graus de mal-estar ou desconforto com um excessivo estado de excitação, sentimentos de medo, incerteza ou apreensão (Carvalho, Bertolini, Milani \& Martins, 2015).

De acordo com American Psychiatric Association (2014), o TA se diferencia do medo ou da ansiedade adaptativos por serem excessivos ou persistirem além de períodos apropriados ao nível de desenvolvimento. Eles diferem do medo ou da ansiedade provisórios, com frequência induzidos por estresse, por serem persistentes, embora o critério para a duração seja tido como um guia geral, com a possibilidade de algum grau de flexibilidade, sendo às vezes de duração mais curta.

O ingresso no ensino superior e a vivência acadêmica caracterizam um marco significativo que coincide a um período do desenvolvimento psicossocial marcado por mudanças importantes, como o período de explorações, a idade das possibilidades e instabilidades. Há ocorrência de mudanças ambientais, como na rotina e nos sistemas de suporte social, além disso, o afastamento do jovem do ambiente familiar e da rede social anterior ao ensino superior. Desse modo, esse novo cenário pode gerar instabilidade, apresentar medo, preocupação, dúvida e ansiedade (Osse \& Costa, 2011).

O TA é classificado como um dos três problemas mais comuns no que se refere à saúde mental no mundo contemporâneo, podendo atingir o grau de patologia, quando interfere de forma incisiva na rotina dos indivíduos, gerando um comprometimento negativo na vida pessoal, social e profissional (Dos Santos \& Pires, 2016). A prevalência do TA em estudantes universitários, alcança a preocupante marca de 11,6 \%, apresentando os primeiros sinais precocemente por volta dos onze anos de idade, sendo predominante no sexo feminino (Nobile, Garcia \& Silva, 2017).

Os fatores associados ao risco de ansiedade estão significativamente mais presentes entre os estudantes que apresentaram relacionamentos interpessoais insatisfatório; além disso, fatores como insônia, não fazer atividade física e preocupação demasiada, também apresentam associação significativa com um quadro de ansiedade em sua condição patológica, apresentando-se de forma mais frequente e intensa, com sintomas que podem causar grande sofrimento e prejuízo na vida cotidiana, como evasão escolar, abandono de emprego e abuso de substâncias (Leão, Gomes, Ferreira \& Cavalcanti, 2018). 
A partir do conjunto legislativo do Sistema Único de Saúde através das Leis 8080/1990 e 8142/1990, e Lei Federal 10.216/2001 que dispõe sobre a proteção e os direitos das pessoas portadoras de transtornos mentais e redireciona o modelo assistencial em saúde mental; possibilitou e estabeleceu diretrizes para uma assistência à saúde mental centrada em recursos comunitários e em um atendimento extra-hospitalar. A Política Nacional de Saúde Mental, vigente no Brasil, desenvolvida pelo Ministério da Saúde, objetiva reduzir progressivamente os leitos em hospitais psiquiátricos, expandindo, qualificando e fortalecendo a rede extra-hospitalar através da implementação de serviços substitutivos, baseados também em trabalho multidisciplinar (Brasil, 2005).

Nessa perspectiva se torna necessário estudar a ansiedade em universitários, desse modo, o objetivo do estudo foi traçar o perfil dos níveis de ansiedade em estudantes universitários de um Centro Universitário da cidade de Teresina, Piauí.

\section{Metodologia}

O estudo foi realizado a partir de uma análise descritiva, com abordagem quantitativa. Essa metodologia tem como principal objetivo descrever características de determinada população ou fenômeno o estabelecimento de relações entre as variáveis. Uma de suas características mais significativas está na utilização de técnicas padronizada de coleta de danos. Além disso, a abordagem metodológica permite o emprego de instrumentos estatísticos sobre os dados (Raupp \& Beuren, 2006).

Os dados foram coletados por conveniência de estudantes dos cursos do âmbito da saúde em um Centro Universitário de Teresina-PI. A coleta de dados ocorreu nos horários da jornada acadêmica dos respectivos universitários. A amostra foi composta por 165 sujeitos sendo 78 do sexo feminino e 87 do sexo masculino.

Os critérios de inclusão do estudo compreenderam os alunos que estavam regularmente matriculados na instituição de ensino, ter idade mínima de 18 anos e máxima de 40 anos, independente da raça e classe social e que assinaram o Termo de Consentimento Livre e Esclarecido (TCLE). O critério de exclusão foi: questionários respondidos de maneira incompleta.

Em relação ao instrumento para a coleta dos dados, para descrição de identificação e dados antropométricos se utilizou questionário de própria autoria; e para avaliação do nível de ansiedade se utilizou o Inventário de Ansiedade de Beck (BDI), com tradução e adaptação brasileira de Cunha (2001), instrumento esse que mede e classifica a intensidade da ansiedade. O respectivo instrumento é composto por 21 itens, com alternativas de respostas, variando entre nada, um pouco, moderadamente e gravemente. A classificação recomendada para o nível de ansiedade segue os seguintes critérios de pontuação, mensurados de acordo com o escore presente em cada resposta do instrumento.

Quadro 1. Classificação dos níveis de ansiedade conforme o Inventário de Ansiedade de Beck (BDI).

\begin{tabular}{|c|c|}
\hline \multicolumn{2}{|c|}{ Níveis de ansiedade } \\
\hline Ansiedade mínima & $(0-7)$ \\
Ansiedade leve & $(8-15)$ \\
Ansiedade moderada & $(16-25)$ \\
Ansiedade grave & $(26-63)$ \\
\hline
\end{tabular}

Fonte: Cunha (2001).

A síntese dos dados foi realizada mediante tabulação com dupla digitação e dupla checagem utilizando o Software Microsoft Office Excel. Para análise, utilizando os recursos do mesmo programa, procedeu-se a estatística descritiva em frequências relativas, percentuais, médias, valor máximo e mínimo e desvio padrão. Para diagnosticar o perfil de ansiedade foi utilizado o percentual de frequências juntamente com os scores dos instrumentos. 
Este estudo foi executado com base no banco de dados do projeto de pesquisa "Associação entre insatisfação com a imagem corporal e esquemas de gênero do autoconceito em adultos jovens", que foi encaminhado ao Comitê de Ética, via Plataforma Brasil, em atendimento a resolução do CNS 486/12 que regulamenta a pesquisa em seres humanos e foi aprovado pelo comitê de ética da Universidade Católica de Brasília, com o CAAE: 96216318.1.0000.5602 e Parecer nº 3.099.282.

\section{Resultados}

A amostra foi composta por 165 universitários sendo 87 do sexo masculino e 78 do sexo feminino do Centro Universitário Santo Agostinho - UNIFSA, escolhidos por conveniência. Através da análise estatística como idade média do sexo feminino 22,6 $\pm 4,9$, quanto ao sexo masculino, a média de 22,1 $\pm 4,4$ anos, escolhidos por conveniência do Curso de educação Física.

Tabela 1. Analise estatística da idade e características antropométricas da amostra por sexo.

\begin{tabular}{|c|c|c|c|c|c|c|c|c|}
\hline \multirow[b]{2}{*}{ Variáveis } & \multicolumn{4}{|c|}{ Feminino $(n=78)$} & \multicolumn{4}{|c|}{ Masculino $(n=87)$} \\
\hline & Média & $\begin{array}{l}\text { Desvio } \\
\text { Padrão }\end{array}$ & $\begin{array}{l}\text { Valor } \\
\text { Máximo }\end{array}$ & $\begin{array}{l}\text { Valor } \\
\text { Mínimo }\end{array}$ & Média & $\begin{array}{l}\text { Desvio } \\
\text { Padrão }\end{array}$ & $\begin{array}{l}\text { Valor } \\
\text { Máximo }\end{array}$ & $\begin{array}{l}\text { Valor } \\
\text { Mínimo }\end{array}$ \\
\hline Idade(anos) & 22,6 & 4,9 & 40 & 18 & 22,1 & 4,4 & 40 & 18 \\
\hline Estatura(m) & 1,65 & 0,09 & 1,9 & 1,5 & 1,71 & 0,09 & 1,97 & 1,53 \\
\hline Massa Corporal $(\mathrm{kg})$ & 64,63 & 11,51 & 99,3 & 45 & 71,72 & 14,66 & 120 & 45 \\
\hline IMC $\left(\mathrm{kg} / \mathrm{m}^{2}\right)$ & 23,51 & 2,90 & 31,02 & 18,07 & 24,50 & 4,27 & 37,04 & 10,65 \\
\hline
\end{tabular}

Kg: Quilograma; m: metro; IMC: Índice de Massa Corpórea. Fonte: Dados da Pesquisa (2021).

Diante análise da estatura, a média de 1,65 centímetros no sexo feminino, sendo 1,5 metro mínima e 1,9 metro máxima, já no sexo masculino, a média de 1,71 metro, sendo a mínima de 1,53 metro e máxima de 1,97 metro. Em relação a massa corporal do sexo feminino, a média foi de $64,63 \mathrm{~kg}$, sendo o valor mínimo de $45 \mathrm{~kg}$ e máximo de $99,3 \mathrm{~kg}$; e no masculino a média de 71,22, sendo o valor máximo de $120 \mathrm{~kg}$ e mínimo $45 \mathrm{~kg}$. O IMC do sexo feminino tem média de $23,51 \mathrm{~kg} / \mathrm{m}^{2}$, sendo o valor mínimo de $18,07 \mathrm{~kg} / \mathrm{m}^{2}$ e máximo de $31,02 \mathrm{~kg} / \mathrm{m}^{2}$, já o do sexo masculino a média de $24,50 \mathrm{~kg} / \mathrm{m}^{2}$, sendo o valor mínimo de $10,65 \mathrm{~kg} / \mathrm{m}^{2}$ e máximo de $37,04 \mathrm{~kg} / \mathrm{m}^{2}$.

Dentre a análise de outras variáveis amostrais, podemos observar que o sexo masculino em relação ao sexo feminino obteve-se maior prevalência perante a prática de atividade física, como explicitado na Tabela 2 a seguir.

Tabela 2. Analise da frequência absoluta e percentagem dos praticantes de exercício físicos do sexo masculino e feminino.

\begin{tabular}{cccc}
\hline & \multicolumn{2}{c}{ Variáveis } & \\
\hline Sexo & Atividade Física & Frequência & Percentual \\
\hline Masculino & Sim & 39 & $24 \%$ \\
Masculino & Não & 48 & $29 \%$ \\
Feminino & Sim & 23 & $14 \%$ \\
Feminino & Não & 55 & $33 \%$ \\
\hline
\end{tabular}

Fonte: Dados da Pesquisa (2021). 
Quanto a tabela anterior podemos observar que o número de participantes do sexo masculino que praticam atividade física é de 39 (24\%), inferior aos que não praticam 48 (29\%), enquanto que, número do sexo feminino que pratica é de 23 $(14 \%)$, sendo novamente inferior a quem não pratica 55 (33\%).

Tabela 3. Classificação e análise dos níveis de ansiedade dos acadêmicos do sexo masculino e feminino.

\begin{tabular}{|c|c|c|c|c|c|}
\hline \multicolumn{6}{|c|}{ Níveis de Ansiedade } \\
\hline Níveis & Sexo & Frequência & Percentual & Frequência Total & Percentual Total \\
\hline \multirow{2}{*}{ Mínimo } & Masculino & 4 & $2,4 \%$ & \multirow{2}{*}{4} & \multirow{2}{*}{$2,4 \%$} \\
\hline & Feminino & 0 & $0,0 \%$ & & \\
\hline \multirow{2}{*}{ Leve } & Masculino & 7 & $4,2 \%$ & \multirow{2}{*}{9} & \multirow{2}{*}{$5,5 \%$} \\
\hline & Feminino & 2 & $1,3 \%$ & & \\
\hline \multirow{2}{*}{ Moderado } & Masculino & 26 & $15,8 \%$ & \multirow{2}{*}{48} & \multirow{2}{*}{$29,1 \%$} \\
\hline & Feminino & 22 & $13,3 \%$ & & \\
\hline \multirow{2}{*}{ Grave } & Masculino & 50 & $30,3 \%$ & \multirow{2}{*}{104} & \multirow{2}{*}{$63 \%$} \\
\hline & Feminino & 54 & $32,7 \%$ & & \\
\hline \multirow{2}{*}{ Total } & Masculino & 87 & \multirow{2}{*}{$100 \%$} & \multirow{2}{*}{165} & \multirow{2}{*}{$100 \%$} \\
\hline & Feminino & 78 & & & \\
\hline
\end{tabular}

Fonte: Dados da Pesquisa (2021).

Diante a classificação dos níveis de ansiedade entre os acadêmicos, 4 (2,4\%) apresentaram a classificação mínima, sendo todos esses do sexo masculino, 9 apresentaram nível leve, sendo 7 (4,2\%) do sexo masculino e 2 (1,3\%) do sexo feminino, 48 apresentaram nível moderado, sendo 26 (15,8\%) do sexo masculino e $22(13,3 \%)$ do sexo feminino e 104 apresentaram nível considerado grave, sendo 50 (30,3\%) do sexo masculino e 54 (32,7\%) do sexo feminino. Em todos os níveis, com exceção do nível grave de ansiedade, o sexo masculino prevaleceu. A prevalência dos níveis grave esteve mais associada ao sexo feminino.

Os escores de classificação da ansiedade são resultados da soma dos escores dos itens individuais permitindo a classificação dos níveis de ansiedade explícitos na tabela anterior. Diante disso, o quadro a seguir representa os escores graves na qual revela a prevalência dos sintomas comuns a ansiedade no sexo masculino e feminino diante do instrumento BDI. 
Quadro 2. Sintomas que apresentaram maior prevalência nos casos de ansiedade grave segundo o Manual do Inventário de Beck diferenciados pelo sexo.

\begin{tabular}{|lccc|}
\hline & & Casos Graves de Ansiedade & \\
\hline \multirow{3}{*}{ Sexo Masculino } & Descrição & Frequência \\
\hline & Beck 5 & Medo que aconteça o pior. & 32 \\
& Beck 10 & Nervoso. & 21 \\
& Beck 2 & Sensação de calor. & 18 \\
& Beck 16 & Incapaz de relaxar. & 15 \\
& Beck 5 & Medo de morrer. & 13 \\
Sexo Feminino & Medo que aconteça o pior. & 24 \\
& Beck 10 & Nervoso. & 22 \\
& Beck 4 & Incapaz de relaxar. & 21 \\
& Beck 2 & Sensação de calor. & 21 \\
\hline
\end{tabular}

Fonte: Dados da Pesquisa (2021).

Dentre os sintomas elencados pela BDI, os que apresentaram maior prevalência em ambos os sexos, foram Beck 5 (Medo que aconteça o pior) e Beck 10 (Nervoso). Ainda são evidentes nos dois sexos os sintomas de "Incapaz de relaxar" Beck 4 e "Sensação de calor" - Beck 2. Entre os cinco principais sintomas de ansiedade grave relacionados aos sexos, diferemse apenas os sintomas de "Medo de morrer" - Beck 16 amostrados no sexo masculino e "Palpitação ou aceleração do coração" - ao sexo feminino.

\section{Discussão}

A ansiedade apesar de ser uma característica comum aos seres, em proporções elevadas torna-se patológica. Em relação a ocorrência dessa no estudo, observou-se que os escores de ansiedade grave foram prevalentes em 104 estudantes, compreendendo $63 \%$ dos casos. Isso elucida que a ansiedade grave é uma condição a maior parte dos alunos entrevistados.

Costa, Branco, Vieira, Souza e Silva (2019) corroboram com o achado descrito no parágrafo anterior, em seu estudo transversal de base populacional, na qual foi observado alta prevalência de transtorno de ansiedade na população investigada: 27,4\% ( $\mathrm{n}=536$ ), porém na pesquisa de Munir Gomes, Pereira Junior, Viana Cardoso e Augusto da Silva (2020) dos 378 alunos universitários entrevistados, 151 (39,9\%) apresentaram escore de classificação para casos de ansiedade e de somatização. Costa et al. (2020), verificaram a presença de sintomas ansiosos em 33,6\% dos estudantes pesquisados.

Nogueira et al. (2021) destacaram em seu estudo transversal, além da maior prevalência a maior propensão do sexo feminino a desenvolver casos graves de ansiedade; o que corrobora o estudo transversal analítico de Leão, Gomes, Ferreira e Cavalcanti (2018) na qual destacaram índices mais elevados de ansiedade no sexo feminino. Já nos resultados do presente estudo, demonstram que os casos dos níveis de mínimo a moderado houve prevalência do sexo masculino.

No entanto, as formas graves de ansiedade, representaram 63\%, existindo assim, uma discreta maioria do sexo feminino, com o número de 54 casos, 32,7\% do número total. A pesquisa de Melo, Peixoto, Oliveira e Bizarro (2012) reforça o 
nivelamento entre os sexos na demonstração de ansiedade grave, em que afirma em seu estudo que a caraterização dos níveis de ansiedade em relação ao sexo não apresentou significância estatística.

Costa, Branco, Vieira, Souza e Silva (2019) descrevem que os participantes da pesquisa apresentaram idade entre uma idade 18 e 35 anos, encontraram-se mais vulneráveis para a ansiedade, por estarem em busca do engajamento no mercado de trabalho, reconhecimento profissional e, muitas vezes, passando pela experiência da maternidade e formação de família. Pode ainda, ser um reflexo originado desde a infância e juventude, além disso, podendo levar a consequências como prejuízo funcional e social, afetando a vida quando adultos.

Montiel, Bartholomeu, Machado e Pessotto (2014) apresentaram em seu estudo, utilizando instrumento de pesquisa similar, cujo objetivo é a caracterização dos sintomas no transtorno de ansiedade, que na amostra tiveram como resultados mais significativos: a incapacidade de relaxar, medo que aconteça o pior, ser nervoso, sensações de sufocação, tremor nas mãos, medo de perder o controle, medo de morrer, assustado e suor não devido ao calor. No contexto da sintomatologia observada, pode se considerar um estado emocional com componentes psicológicos e fisiológicos, sendo que esses, tendem a ocasionar desajustes e complicações pessoais e sociais como observado no tempo de duração das crises de ansiedade, ocasionando idas frequentes a especialidades médicas e serviços de saúde.

No entanto, no presente estudo, dentre os principais sintomas das formas graves de ansiedade, quatro desses são comuns a ambos os sexos: medo que aconteça o pior (beck 5); nervoso (beck 10), incapaz de relaxar (beck 4) e sensação de calor (beck 2). Entre o sexo feminino, ainda foi evidenciado mais um sintoma relevante aos casos graves: palpitação ou aceleração do coração (beck 7); e entre o sexo masculino, outro sintoma notório em casos graves foi: medo de morrer (beck 16).

Cardozo, Gomes, Fan e Soratto (2016) demostram em sua pesquisa de abordagem quali-quantitativa, descritiva e exploratória, os sintomas entre os 27 estudantes de nível superior os sintomas de ansiedade comuns durante certo momento do curso, na qual, os sintomas apresentados em maior evidencia foram: medo e insegurança. Santos e Galdeano (2009) em estudo prospectivo, transversal, descritivo-exploratório, com abordagem quantitativa com 49 alunos do curso de enfermagem de uma instituição de ensino superior, caracterizaram os sintomas de ansiedade em maior evidência entre os alunos, acompanhado de medo, nervosismo, preocupação e angústia.

Em relação aos fatores associados o fator idade, Santos e Galdeano (2009) revelam ainda em seu estudo que, a maior incidência de ansiedade com sintomas graves em estudante com idade superior a 30 anos; ao contrário do estudo observacional, quantitativo de Toti, Bastos e Rodrigues (2019), verificou-se uma associação entre sintomas graves de ansiedade em estudantes mais jovens.

No tocante à prática dos exercícios os autores anteriores observaram que os resultados para praticantes de atividades físicas no lazer, de forma regular, com mais de 150 minutos semanais, apresentaram menor probabilidade de ter depressão e ansiedade; assim como destacado por Leão, Gomes, Ferreira e Cavalcanti (2018) que não fazer atividade física regulares apresentaram associação significativa com um quadro de ansiedade.

Sacramento, Anjos, Barbosa, Tavares e Dias (2021) ressaltam que outros fatores podem explicar, em parte, o índice elevado de ansiedade entre estudantes universitários, dentre eles, está a influência hormonal, posto que, a testosterona pode ter benefícios protetores contra a ansiedade a depressão, além disso, de acordo com Nogueira et al. (2021), as alterações hormonais próprias do sexo feminino que contribuem para esse perfil de ansiedade grave. Leão, Gomes, Ferreira e Cavalcanti (2018) elencam também dentre as alterações orgânicas que contribuem para a disposição da ansiedade em níveis patológicos, o sono e repouso prejudicado caracterizado pela insônia. 


\section{Conclusão}

No presente estudo, verificou-se que a maioria dos universitários apresentaram níveis graves de ansiedade, observando discreta maioria do índice grave relacionado ao sexo feminino. Além disso, ao correlacionar com o contexto da prática de atividade física, observou-se que os níveis de ansiedade foram maiores no sexo feminino que não aderem a atividade física regular em seus hábitos. Outrossim, na caracterização dos sintomas graves entre os participantes, existiu discreta diferença entre a percepção dos sintomas quando relacionadas ao sexo.

Nesse sentido, o estudo revela um contexto de ansiedade entre os alunos de uma instituição de ensino superior, que contribui para a compreensão e formação de condutas relevantes para transformação desse âmbito. Dessa forma, deve-se repensar sobre o processo de formação desde o ingresso até a sua conclusão, buscar por uma formação assistida, na qual o estudante tenha a possibilidade de ser atendido em suas necessidades pedagógicas e emocionais. Salienta-se, ainda, a importância de adoção, pela instituição formadora, de medidas voltadas para identificação de fatores de risco para a ansiedade exacerbada nos estudantes, revisão das metodologias de ensino adotadas, outrossim, viabilizar estratégias, de modo a minimizar as repercussões da ansiedade patológica.

\section{Referências}

American Psychiatric Association. (2014). DSM-5: Manual diagnóstico e estatístico de transtornos mentais. Artmed Editora.

Binelli, C., Ortiz, A., Muñiz, A., Gelabert, E., Ferraz, L., Crippa, J. A. S., \& Martín-Santos, R. (2012). Eventos negativos na infância e ansiedade social em estudantes universitários. Brazilian Journal of Psychiatry, 34(1), 69-80. https://doi.org/10.1590/S1516-44462012000500006.

Brasil, M. D. S. Ministério da Saúde Lei no 10.216 2001. Ministério da Saúde; 2005. http://www.saude.gov.br

Cardozo, M. Q., Gomes, K. M., Fan, L. G., \& Soratto, M. T. (2016). Fatores associados à ocorrência de ansiedade dos acadêmicos de Biomedicina. Saúde e Pesquisa, 9(2), 251-262. DOI: https://doi.org/10.17765/2176-9206.2016v9n2p251-262

Carvalho, E. A. D., Bertolini, S. M. M. G., Milani, R. G., \& Martins, M. C. (2015). Índice de ansiedade em universitários ingressantes e concluintes de uma instituição de ensino superior. Ciênc. cuid. saúde, 1290-1298. https://doi.org/10.4025/cienccuidsaude.v14i3.23594

Costa, C. O. D., Branco, J. C., Vieira, I. S., Souza, L. D. D. M., \& Silva, R. A. D. (2019). Prevalência de ansiedade e fatores associados em adultos. Jornal Brasileiro de Psiquiatria, 68(2), 92-100. 10.1590/0047-2085000000232.

Costa, D. S. D., Medeiros, N. D. S. B., Cordeiro, R. A., Frutuoso, E. D. S., Lopes, J. M., \& Moreira, S. D. N. T. (2020). Sintomas de Depressão, Ansiedade e Estresse em Estudantes de Medicina e Estratégias Institucionais de Enfrentamento. Revista Brasileira de Educação Médica, 44(1). DOI: https://doi.org/10.1590/1981-5271v44.1-20190069.

Cunha, J. A. (2001). Manual da versão em português das Escalas Beck. casa do psicólogo, 256.

Dos Santos, L. F., \& Pires, E. U. (2016). Fobia social em adolescentes: repercussões acadêmicas. Revista de Psicologia da IMED, 8(2), 172-184. https://doi.org/10.18256/2175-5027/psico-imed.v8n2p172-184

Leão, A. M., Gomes, I. P., Ferreira, M. J. M., \& Cavalcanti, L. P. D. G. (2018). Prevalência e fatores associados à depressão e ansiedade entre estudantes universitários da área da saúde de um grande centro urbano do Nordeste do Brasil. Revista brasileira de educação médica, 42(4), 55-65. http://dx.doi.org/10.1590/1981-52712015v42n4RB20180092.

Melo, W. V., Peixoto, M., Oliveira, A., \& Bizarro, L. (2012). Avaliação da ansiedade e do processamento da atenção em universitários através da tarefa de Stroop Emocional. Revista Brasileira de Terapia Comportamental e Cognitiva, 14(2), 23-35. ISSN 1517-5545

Montiel, J. M., Bartholomeu, D., Machado, A. A., \& Pessotto, F. (2014). Caracterização dos sintomas de ansiedade em pacientes com transtorno de pânico. Boletim Academia Paulista de Psicologia, 34(86), 171-185..

Munir Gomes, C. F., Pereira Junior, R. J., Viana Cardoso, J., \& Augusto da Silva, D. (2020). Transtornos mentais comuns em estudantes universitários: abordagem epidemiológica sobre vulnerabilidades. SMAD Revista Electronica Salud Mental, Alcohol y Drogas, 16(1). 10.11606/issn.18066976.smad.2020.157317.

Nobile, G. F. G., Garcia, V. A., \& Silva, A. T. B. (2017). Análise Sequencial dos comportamentos do terapeuta em psicoterapia com universitários com transtorno de ansiedade social. Perspectivas em análise do comportamento, 8(1), 16-31. https://doi.org/10.18761/pac.2016.030

Nogueira, É. G., Matos, N. C. D., Machado, J. N., Araújo, L. B. D., Silva, A. M. T. C., \& Almeida, R. J. D. (2021). Avaliação dos níveis de ansiedade e seus fatores associados em estudantes internos de Medicina. Revista Brasileira de Educação Médica, 45(1). https://doi.org/10.1590/1981-5271v45.1-20200174.

Organização Mundial da Saúde. (2017). Depressão e outros transtornos mentais comuns: estimativas de saúde global (No. WHO / MSD / MER / 2017.2). Organização Mundial da Saúde. 
Research, Society and Development, v. 10, n. 6, e14910615420, 2021

(CC BY 4.0) | ISSN 2525-3409 | DOI: http://dx.doi.org/10.33448/rsd-v10i6.15420

Osse, C. M. C., \& Costa, I. I. D. (2011). Saúde mental e qualidade de vida na moradia estudantil da Universidade de Brasília. Estudos de Psicologia (Campinas), 28(1), 115-122. https://doi.org/10.1590/S0103-166X2011000100012

Raupp, F. M., \& Beuren, I. M. (2006). Metodologia da pesquisa aplicável às ciências. Como elaborar trabalhos monográficos em contabilidade: teoria e prática. Atlas, 76-97.

Sacramento, B. O., Anjos, T. L. D., Barbosa, A. G. L., Tavares, C. F., \& Dias, J. P. (2021). Sintomas de ansiedade e depressão entre estudantes de medicina: estudo de prevalência e fatores associados. Revista Brasileira de Educação Médica, 45(1). https://doi.org/10.1590/1981-5271v45.1-20200394.

Santos, M. D. L. D., \& Galdeano, L. E. (2009). Traço e estado de ansiedade de estudantes de enfermagem na realização de uma prova prática. Revista Mineira de Enfermagem, 13(1), 76-83.

Toti, T. G., Bastos, F. A., \& Rodrigues, P. F. (2019). Fatores associados à ansiedade e depressão em estudantes universitários do curso de educação física. Revista Saúde Física \& Mental. 6(2), 21-30.

Vasconcelos, T. C. D., Dias, B. R. T., Andrade, L. R., Melo, G. F., Barbosa, L., \& Souza, E. (2015). Prevalence of anxiety and depression symptoms among medicine students. Revista Brasileira de Educação Médica, 39(1), 135-142. https://doi.org/10.1590/1981-52712015v39n1e00042014. 